\title{
Video-assisted thoracoscopic right upper sleeve lobectomy after neoadjuvant chemotherapy for central non-small cell lung cancer
}

\author{
Zhuoqi Jia ${ }^{1}$, Weiru Zhou ${ }^{2}$, Shuo Li $^{1}$, Yong Zhang ${ }^{1}$, Guangjian Zhang ${ }^{1}$, Junke Fu ${ }^{1}$ \\ ${ }^{1}$ Department of Thoracic Surgery, First Affiliated Hospital of Xi'an Jiaotong University, Xi'an, China; ${ }^{2}$ Department of Nuclear Medicine, Xi'an \\ Gaoxin Hospital, Xi'an, China \\ Correspondence to: Zhuoqi Jia, MD. Department of Thoracic Surgery, First Affiliated Hospital of Xi'an Jiaotong University. 277\#, West Yanta Road, \\ Xi'an 710061, China. Email: gosnow@163.com.
}

\begin{abstract}
Sleeve lobectomy is an uncommon thoracic surgical procedure. Nonetheless, it is an accepted treatment option to avoid pneumonectomy in patients with centrally, locally advanced non-small cell lung cancer. In appropriately selected patients, the outcome of sleeve lobectomy is equivalent to pneumonectomy with comparable recurrence rates, with less perioperative morbidity, improved functional outcomes, and superior quality of life post-surgery. According to the National Comprehensive Cancer Network (NCCN) or Chinese Society of Clinical Oncology (CSCO) guidelines, neoadjuvant chemotherapy has become the standard treatment regimen in patients with stage N2 non-small cell lung cancer, despite the high risk of associated complications, due to its proven efficacy in improving prognosis in these patients. Here we report a case of a central squamous carcinoma in the upper lobe of the right lung. Bronchoscopy revealed rough and uplifted mucosa in the right upper bronchus and a narrow lumen. The patient was diagnosed with squamous carcinoma of the right upper lobe with subcarinal lymph node metastasis, and no distant metastasis (clinical stage cT4N2M0, IIIB). The Multi-Disciplinary Treatment (MDT)team recommended 2 cycles of neoadjuvant chemotherapy. Although the size of the tumor had reduced significantly after the second cycle, as observed on the CT images, it was still close to orifice of the right upper bronchus. So our surgical team performed a right upper sleeve lobectomy with 3-port thoracoscope. The patient recovered very well after the procedure. Our first experience of bronchial reconstruction after neoadjuvant chemotherapy showed no increased morbidity and mortality, making it a technically safe and feasible approach.
\end{abstract}

Keywords: Video-assisted thoracoscopic surgery (VATS); sleeve lobectomy (SL); lymph nodes dissection; neoadjuvant chemothrapy

Received: 17 April 2020; Accepted: 28 January 2021; Published: 25 August 2021.

doi: $10.21037 /$ ccts-20-85

View this article at: http://dx.doi.org/10.21037/ccts-20-85

\section{Introduction}

Surgical procedures for the treatment of lung cancer started 80 years ago, when Graham performed the first successful pneumonectomy in 1933. Nowadays, the standard surgical procedure to treat non-small cell lung cancer (NSCLC) is anatomical lobectomy with systemic lymph nodes dissection (1). Such an approach is not feasible for cases with central lung cancer with invasion to the bronchial orifice, as lobectomy cannot completely remove the tumor. Besides, these patients have poor lung function and cannot tolerate pneumonectomy. Hence, sleeve lobectomy (SL) should be considered as the treatment of choice in these patients. This procedure dates back to 1947, when Clement Price Thomas successfully performed the first bronchus sleeve resection in London, in a patient with right bronchoadenoma (2). This procedure reportedly does not increase the rate of postoperative complications and mortality, which has resulted in the majority of thoracic surgeons to accept and use it in clinic. Traditional SL is an open thoracic surgery, but with the rapid development of video-assisted thoracoscopic surgery in recent years, some surgeons have 

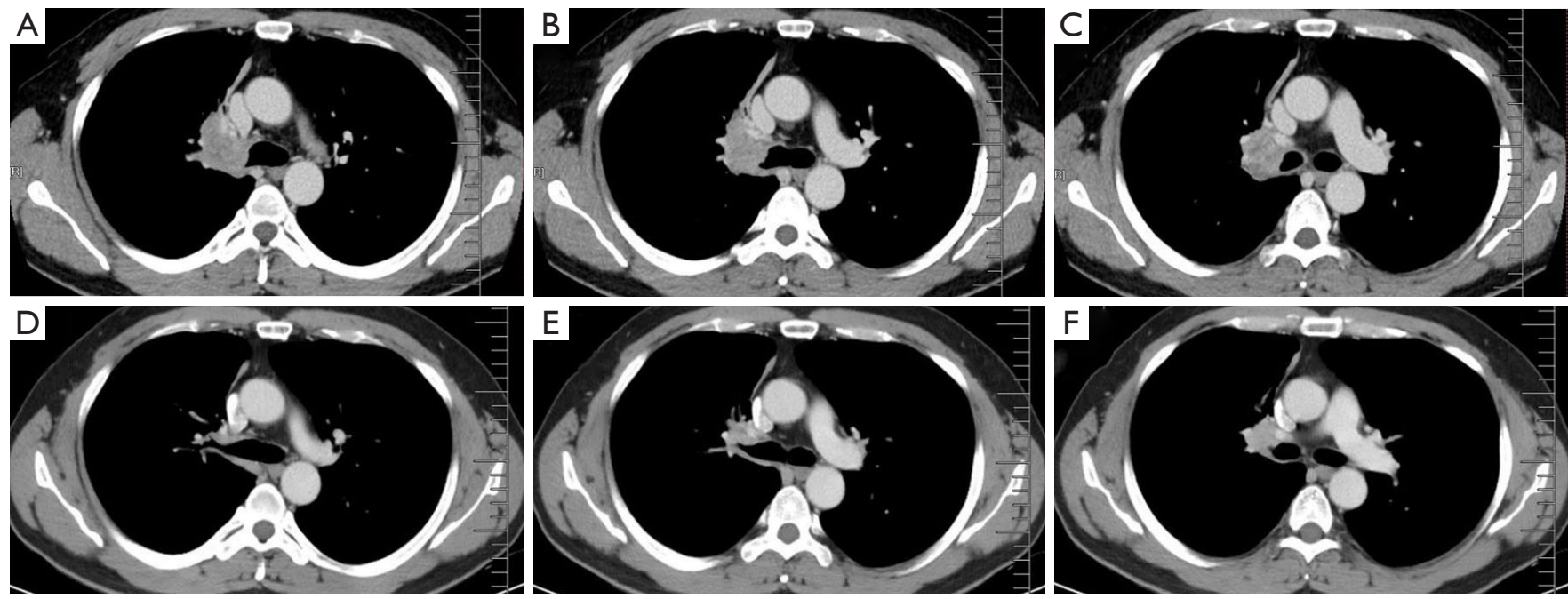

Figure 1 Chest CT before (A,B,C) and after neoadjuvant chemotherapy (D,E,F).

performed SL using a video-assisted thoracoscopic surgery (VATS) approach. Previous studies have shown that the major surgical steps of SL are complicated and difficult, and the clinical efficacy and long-term outcomes of this treatment in patients with NSCLC are still controversial (3-5). Here we present a case of VATS right upper sleeve lobectomy for NSCLC after neoadjuvant chemotherapy.

\section{Clinical summary}

A 55-years-old male, complained of cough and shortness of breath for two months. Chest computerized tomography showed a neoplastic growth in the right upper lobe invading the orifice (Figure 1). Bronchoscopy revealed rough and uplifted mucosa in the right upper bronchus and a narrow lumen (Figure 2). Pathological assessment of the biopsy taken during bronchoscopy reported the lesion as squamous carcinoma. On PET CT images we observed right lung tumor, with maximum standard unit value (SUVmax) of 9.8. The subcarinal lymph nodes had an SUVmax of 2.8, which indicated the possibility of metastasis. Based on the pathological and imaging findings, the patient was diagnosed with right upper lobe squamous carcinoma of the lung with subcarinal lymph node metastasis. No distant metastasis was found on whole body scan. As such, the tumor was at clinical stage cT4N2M0 (IIIB). The multidisciplinary team (MDT) recommended two cycles of neoadjuvant chemotherapy consisting of cisplatin and gemcitabine (cisplatin $25 \mathrm{mg} / \mathrm{m}^{2}$ days $1-3$ and gemcitabine $1,000 \mathrm{mg} / \mathrm{m}^{2}$ days 1 and 8 in a 21 -day of each cycle). The patient experience mild gastrointestinal side effects after chemotherapy, but no severe adverse event was reported. After neoadjuvant chemotherapy, chest CT scan revealed significant reduction in the size of the tumor, which was still close to the orifice of the right upper bronchus (Figure 1D,E,F) and not change in the subcarinal lymph nodes. In the second meeting of the MDT team on the treatment plans for this patient, most members agreed with radical surgery (sleeve resection or pneumonectomy). So our surgery team planned a VATS sleeve lobectomy of the right upper lobe with lymph node dissection. All procedures performed in this study were in accordance with the ethical standards of the institutional and/or national research committee(s), and with the Helsinki Declaration (as revised in 2013). Written informed consent was obtained from the patient for publication of this case report and accompanying images. A copy of the written consent is available for review by the editorial office of this journal. The IRB approval number was XJTUAF2019LSK-189 (First Affiliated Hospital of Xi'an Jiaotong University).

\section{Surgical techniques}

\section{Anesthesia, positioning, and port placement}

The patient was placed in the left lateral decubitus and fold position. General anesthesia was induced, and the patient was intubated with a double-lumen endobronchial tube for one lung ventilation. We used the standard 3-port approach (Figure 3). Two $10 \mathrm{~mm}$ ports were made, one in the 7 th 

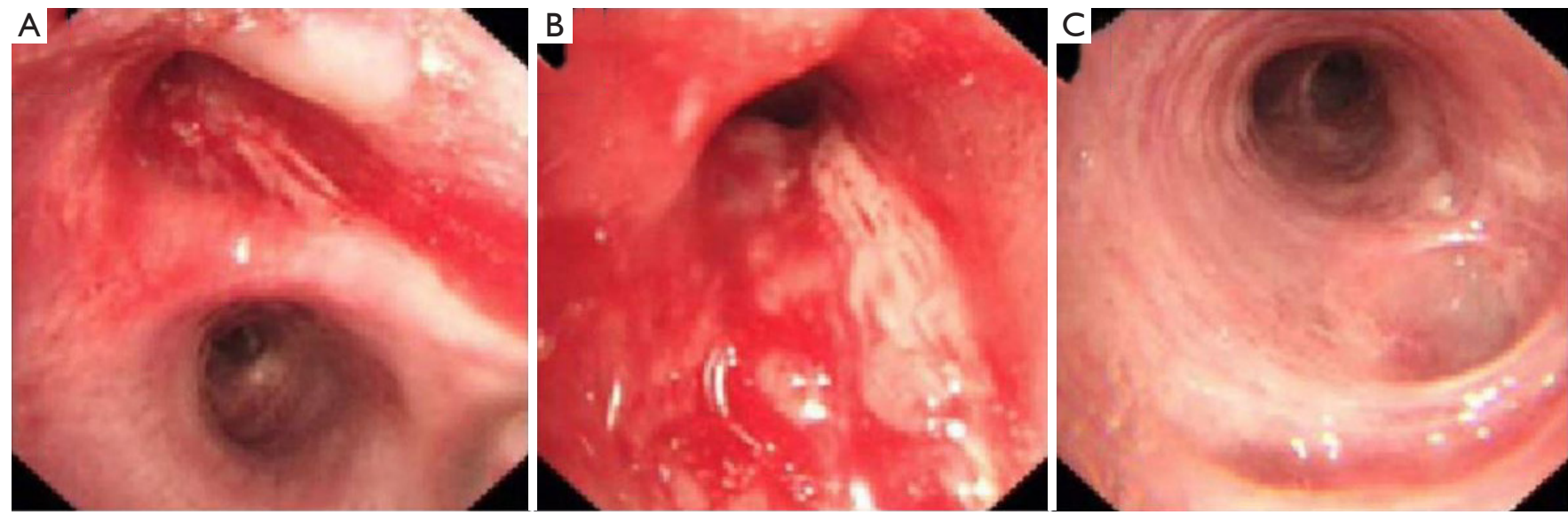

Figure 2 Bronchoscope before chemotherapy. (A) Right main bronchus; (B) right upper bronchus; (C) right intermediated bronchus.

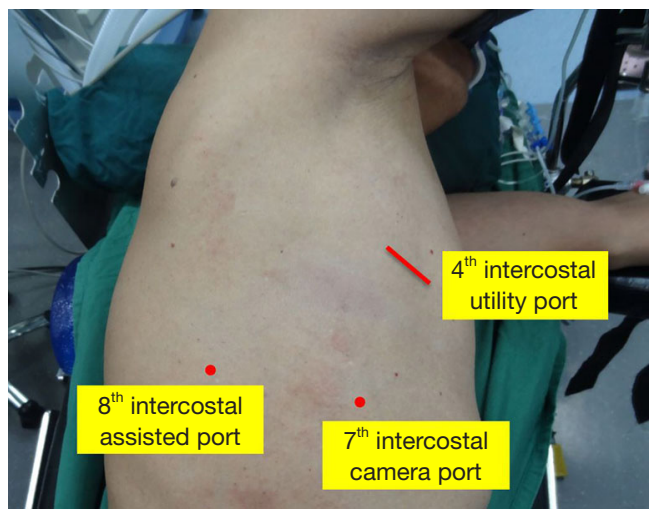

Figure 3 Patient's position and surgery ports placement.

intercostal space on the midaxillary line as the camera port and one at the 8 th intercostal space on the scapular line as assisted port. The utility port was 35 to $45 \mathrm{~mm}$, located in the 4th intercostal space on the anterior axillary line, which was kept open by a plastic wound protector.

\section{Right upper lobectomy with radical mediastinal lymph nodes dissection}

After pleural exploration, to ensure no metastasis to the pleural cavity, the inferior pulmonary ligament was released thoracoscopically. As per the usual protocol used in our hospital, we dissected and severed the superior pulmonary vein first, followed by the superior pulmonary artery branches $\mathrm{A}^{1+3}$ and $\mathrm{A}^{2}$ using an endoscopic straight cutting stapler (Figure 4A,B,C,D,E,F). The interlobar lymph nodes surrounding the right upper bronchus were also dissected at the same time. The hilar, subcarinal, and para-tracheal lymph nodes en-bloc removed by using energy and suction equipment with no-clamping technique (Figure $4 G, H$ ). The major step was to remove the entire subcarinal nodal tissue to expose the borders of the right main bronchus. Dissecting along the right main bronchus should be dome with extra care to avoid energy injury. The azygos vein was dissected and suspended using suture knots to improve the surgical field of vision during bronchus reconstruction.

\section{Bronchial anastomosis}

Before bronchotomy, we dissected the tissues around the right intermediate and main bronchi based on the estimated length of the tumor. By pulling back the tube into the trachea, the anesthetist used an intraoperative bronchoscopy guide to locate the tumor and determine its boundary. We first incised the right intermediate bronchus distally prior to a proximal incision of the right main bronchus (Figure 5). To avoid devascularization and high tension for anastomosis, we recommend a distance of no more than $1-2 \mathrm{~cm}$ between the edge of the bronchotomy and the tumor. After removing the entire tumor macroscopically, biopsies were taken from the margin of the tumor and sent for fast-frozen pathological analysis, as the presence of positive margins indicates further resection of the airway. In our case, the fast-frozen pathological analysis showed no tumor cell indicating a negative tumor margin.

After trimming and aligning the bronchus, we used 3-0 PDS (double needles) to perform a running suture. We started suturing from the deepest area on the posterior walls of the bronchus, which is the junction of the rings and 

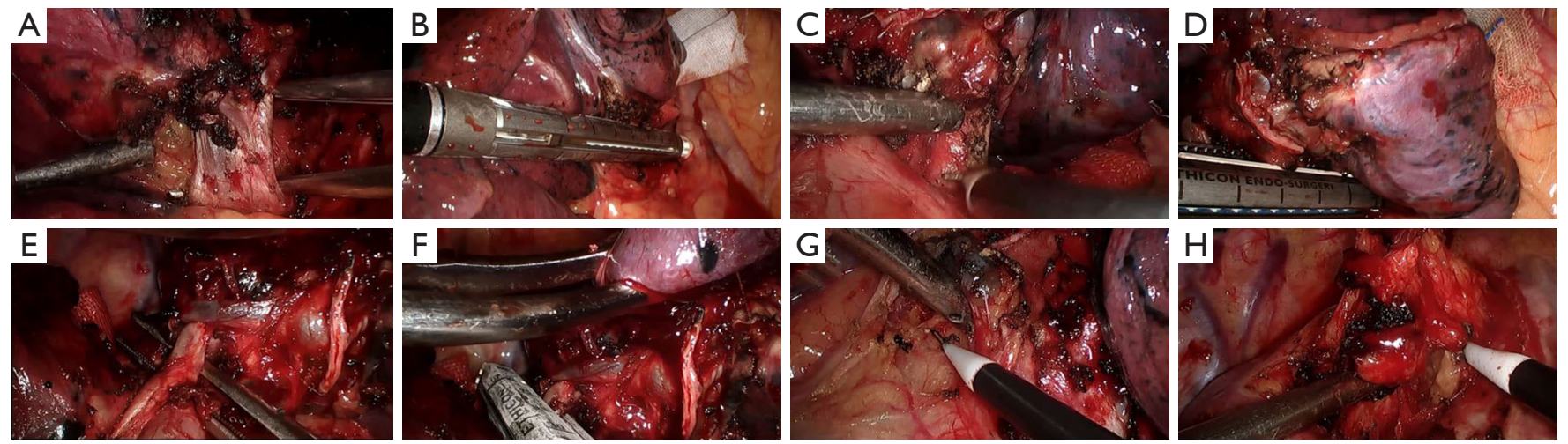

Figure 4 Right upper lobectomy procedures. (A) Dissociated and (B) cut off right superior pulmonary vein; (C) dissociated and (D) cut off superior pulmonary artery branch $\mathrm{A}^{1+3}$; (E) dissociated and $(\mathrm{F})$ cut off superior pulmonary artery branch $\mathrm{A}^{2} ;(\mathrm{G})$ dissection of subcarinal lymph nodes; $(\mathrm{H})$ dissection of para-trachea lymph nodes.
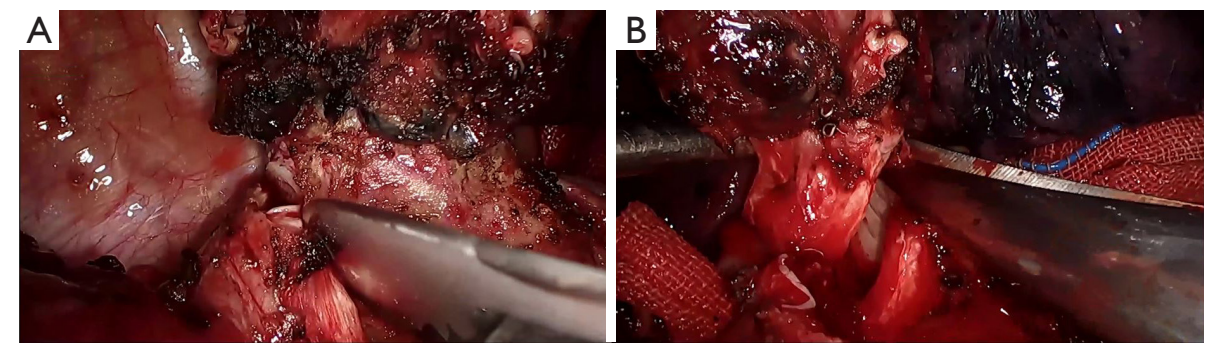

Figure 5 Incised the distal and proximal bronchus by guide of bronchoscopy. (A) Incised the distant right intermediate bronchus; (B) incised the proximal right main bronchus.

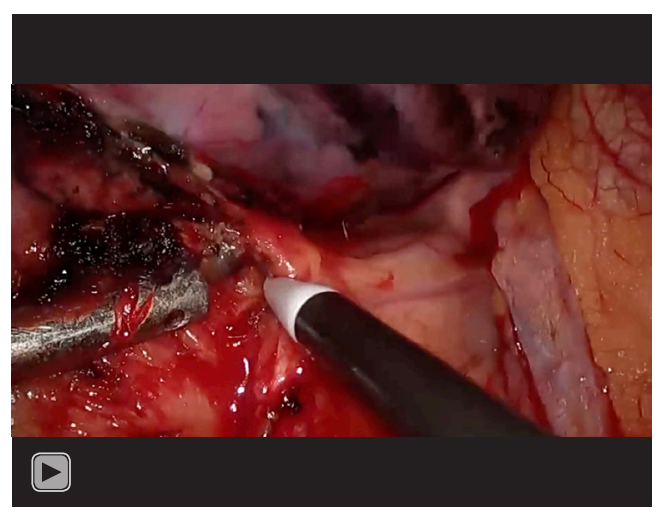

Video 1 VATS right upper sleeve lobectomy after neoadjuvant chemotherapy. VATS, video-assisted thoracoscopic surgery.

membranes. The stiches were about $2 \mathrm{~mm}$ apart. It is crucial that a surgical assistant should maintain a certain tension on the knots to prevent them from coming loose. The front needle was used to suture a third of the circumference of the bronchus first before switching to the posterior needle to continue suturing the side wall for another third of the circumference. The last third was sutured by using the front needle to perform. All knots were tied externally (Video 1). In this procedure, the surgeon should pay extra attention to the direction of suturing and pulling the needle to avoid wrapping the knots. After finishing the anastomosis, the anesthesiologist expanded the lung under a pressure of 20-30 $\mathrm{cm} \mathrm{H}_{2} \mathrm{O}$ to check for any air leak from the site of anastomosis. The mediastinal pleura could be used to wrap the suture the anastomosis if necessary.

\section{Postoperation management}

The operation time was $185 \mathrm{~min}$, and the perioperative blood loss was $175 \mathrm{~mL}$. The number of lymph nodes dissected was 20 , only $1 / 10$ hilar lymph nodes were reported positive. In the first 3 days after the operation, slight air leak was observed in the drainage tube, which stopped by day 
4. Drainage tube was removed on day 6, when chest X-ray showed satisfied lung re-expansion. There were no other severe complications. The final pathology report indicated the tumor to be squamous carcinoma with pTNM stage ypT2N1M0 (IIB).

\section{Comments}

Sleeve resection is commonly used in the treatment of central lung cancer invading the bronchial orifice or surrounding the main/middle bronchi, benign diseases such as low-grade malignant tumors, airway stenosis, or removal of para-bronchial metastatic lymph nodes invading the tracheal wall (6). There are two basic principles for sleeve lobectomy: (I) Completely remove all tumor tissues for R0 resection; (II) Preserve the healthy lung tissue as much as possible. Recent retrospective and meta-analysis studies have shown that sleeve lobectomy represents an acceptable therapeutic option for centrally-located lung cancer, which has lower morbidity and mortality than similar other more radical surgical treatments such as pneumonectomy (7).

In 2002, Santambrogio et al. (8) reported the first case of thoracoscopic assisted sleeve lobectomy in a 15 -yearold female with mucoepidermoid carcinoma located in the bronchial orifice of the left lower lobe, with no tumor recurrence after 40 months of follow-up. In 2008, Mahtabifard et al. (9) reported 13 cases of thoracoscopic sleeve lobectomy in central lung cancer and tuberculous, with satisfactory short-term results and low mortality. Other retrospective studies $(10,11)$ have reported on the benefits of video-assisted thoracoscopic multiportal and uniportal VATS lobectomy and sleeve resection compared with those of thoracotomy and lobectomy (especially sleeve resection), including reduced surgical trauma, less incisional pain, lower incidence of postoperative complication, shorter hospital stay, and earlier recovery to start adjuvant therapies $(12,13)$. Yan et al. (14) reported the criteria of the European Society of Thoracic Surgeons (ESTS) consensus on multiportal VATS lobectomy as: "No use of rib-spreading; Utility incision with a maximum length of $8 \mathrm{~cm}$ to deliver the specimen; Individual dissection of the vein, arteries and airway for the lobe; Standard lymph node sampling or dissection", while the consensus on the criteria for uniportal VATS lobectomy as reported by Bertolaccini et al. (15) were reported as: "The maximum size of the incision should be $\leq 4 \mathrm{~cm}$ in the anterior or middle-anterior axillary line; Eligibility for VATS lobectomy should include tumors in T1/T2 and N0/N1 status; Chest wall involvement was not considered an absolute contraindication for VATS lobectomy, whereas a hilar tumor was considered a relative contraindication. The group recommended systematic dissection of all of the ipsilateral lymph nodes as the most appropriate nodal staging procedure.

However, since most thoracic surgeons are concerned about not achieving a negative tumor margin for malignant tumors after performing sleeve resection, and also the technical challenges associated with a minimally invasive approach, this technique has not been widely accepted (16). Techniques of totally video-assisted thoracoscopic bronchus reconstruction have been regarded as very complex and challenging, even for experienced thoracic surgeons (17-19). Our experiences revealed two important points regarding sleeve lobectomy: (I) When dissociating the bronchus, the cut should be more than $2 \mathrm{~cm}$ away from the edge of the tumor to ensure negative margins. Besides, if the two stumps of a bronchus were of different sizes and hard to anastomose tightly, the main bronchus should be incised vertically, while the middle bronchus should be cut diagonally to ensure size-match. (II) During the reconstruction, 3-0 or 4-0 absorbable PDS should be used to perform a running suture. The surgical assistant must tighten the knots continuously and maintain an appropriate tension to prevent the knots to become loose or entangled. All knots should be tied outside the lumen to ensure proper alignment of the two stumps, and a smaller healing scar. The intercostal muscle flap, the pericardial fat pad, or the thymic fat pad should be used to reinforce the site of anastomosis to prevent fistula.

NSCLC patients with stage N2 disease can be treated with neoadjuvant chemotherapy, chemoradiotherapy followed by surgery, or definitive chemoradiotherapy without surgery (20). A number of studies have reported that surgery following neoadjuvant chemotherapy could reduce tumor size and the TNM stage, resulting in better survival rates (21-23). In our case, tissue edema, fibrous connective tissue hyperplasia, and scar formation in the surgical area resulted from the two cycles of neoadjuvant chemotherapy, which caused a certain degree of difficulty during tissue dissection, but did not affect the suturing procedure or the healing rate of the anastomosis. Gonzalez et al. (24) reported 99 consecutive cases of NSCLC, of which 56 underwent sleeve lobectomy after induction therapy (28 patients), after chemotherapy (12 patients), and after radiochemotherapy (16 patients). In their cohort, no significant differences were observed in postoperative 90day mortality (3.6\% vs. $2.8 \%$ ) and morbidity ( $54 \% v s .49 \%)$ 
in patients who had received induction therapy compared to those who had not. The most common complications after sleeve lobectomy are reportedly bronchopleural fistulas with an incidence rate of $1 \%$ to $5 \%(25)$ and the stenosis of the bronchus at the site of anastomosis with an average incidence rate of $1 \%$ to $4 \%$ as reported by Storelli et al. (26). The 5-year survival for NSCLC after sleeve lobectomy has been reported to be between 39\% and 53\% (27).

In conclusion, video-assisted thoracoscopic sleeve lobectomy for NSCLC after neoadjuvant chemotherapy is technically safe and feasible, and has similar mortality and airway complication rates as those reported for thoracotomy. The treatment should be well planned. Studies with larger cohorts should be performed in the future to demonstrate the benefits of this procedure on long-term survival.

\section{Acknowledgments}

Funding: The study was supported by the Institutional Foundation of The First Hospital of Xi'an Jiaotong University (No. 2020ZYTS-21). The video was awarded third prize in the First International Lung Surgery Competition (2019 Masters of Lung Surgery).

\section{Footnotes}

Peer Review File: Available at https://ccts.amegroups.com/ article/view/10.21037/ccts-20-85/prf

Conflicts of Interest: All authors have completed the ICMJE uniform disclosure form (available at https://ccts. amegroups.com/article/view/10.21037/ccts-20-85/coif). The authors have no conflicts of interest to declare.

Ethical Statement: The authors are accountable for all aspects of the work in ensuring that questions related to the accuracy or integrity of any part of the work are appropriately investigated and resolved. All procedures performed in this study were in accordance with the ethical standards of the institutional and/or national research committee(s), and with the Helsinki Declaration (as revised in 2013). Written informed consent was obtained from the patient for publication of this case report and accompanying images. A copy of the written consent is available for review by the editorial office of this journal. The IRB approval number was XJTUAF2019LSK-189 (First Affiliated Hospital of Xi' an Jiaotong University).
Open Access Statement: This is an Open Access article distributed in accordance with the Creative Commons Attribution-NonCommercial-NoDerivs 4.0 International License (CC BY-NC-ND 4.0), which permits the noncommercial replication and distribution of the article with the strict proviso that no changes or edits are made and the original work is properly cited (including links to both the formal publication through the relevant DOI and the license). See: https://creativecommons.org/licenses/by-nc-nd/4.0/.

\section{References}

1. Pearson FG. Thoracic Surgery second edition[M]. Health Science Asia, Elsevier Science, 2002:1005-13.

2. Thomas CP. Conservative resection of the bronchial tree. J R Coll Surg Edinb 1956;1:169-86.

3. Stallard J, Loberg A, Dunning J, et al. Is sleeve lobectomy significantly better than pneumonectomy? Interact Cardiovasc Thorac Surg 2010;11:660-6.

4. Maurizi G, D'Andrilli A, Anile M, et al. Sleeve lobectomy compared with pneumonectomy after induction therapy for non-small-cell lung cancer. J Thorac Oncol 2013;8:637-43.

5. Gómez-Caro A, Boada M, Reguart N, et al. Sleeve lobectomy after induction chemoradiotherapy. Eur J Cardiothorac Surg 2012;41:1052-8.

6. Eichhorn F, Storz K, Hoffmann H, et al. Sleeve pneumonectomy for central non-small cell lung cancer: indications, complications, and survival. Ann Thorac Surg 2013;96:253-8.

7. Kim YT, Kang CH, Sung SW, et al. Local Control of Disease Related to Lymph Node Involvement in Non-Small Cell Lung Cancer After Sleeve Lobectomy Compared With Pneumonectomy. Ann Thorac Surg 2005;79:1153-61; discussion 1153-61.

8. Santambrogio L, Ciofli U, De Simone M, et al. Video-assisted sleeve lobectomy for mucoepidermoid carcinoma of the left lower lobar bronfbus: a case report. Chest.2002;121:635-6.

9. Mahtabifard A, Fuller CB, McKenna RJ Jr. Video-assisted thoracic surgery sleeve lobectomy: a case series. Ann Thorac Surg, 2008;85:S729-32.

10. Shao F, Liu Z, Pan Y, et al. Bronchoplasty using continuous suture in complete monitor view: a suitable method of thoracoscopic sleeve lobectomy for non-small cell lung cancer. World J Surg Oncol 2016;30;14:134.

11. Soultanis KM, Chen Chao M, Chen J, et al. Technique and outcomes of 79 consecutive uniportal video- 
assisted sleeve lobectomies. Eur J Cardiothorac Surg 2019;56:876-82.

12. Swanson SJ, Herndon JE, D'Amico TA, et al. Video assisted thoracic surgery lobectomy: report of CALGB39802--a prospective, multi-institution feasibility study. J Clin Oncol 2007;25:4993-7.

13. McKenna RJ, Houck W, Fuller CB. Video-Assisted Thoracic Surgery Lobectomy: Experience With 1,100 Cases. Ann Thorac Surg 2006;81:421-5.

14. Yan TD, Cao C, D'Amico TA, et al. Video-assisted thoracoscopic surgery lobectomy at 20 years: a consensus statement. Eur J Cardiothorac Surg 2014;45:633-9.

15. Bertolaccini L, Batirel H, Brunelli A, et al. Uniportal video-assisted thoracic surgery lobectomy: a consensus report from the Uniportal VATS Interest Group (UVIG) of the European Society of Thoracic Surgeons (ESTS). Eur J Cardiothorac Surg 2019;56:224-9.

16. Alam NZ, Flores RM. Extended video-assisted thoracic surgery (VATS) lobectomy. Minerva Chir 2016;71:67-71.

17. Huang J, Li J, Qiu Y, et al. Thoracoscopic double sleeve lobectomy in 13 patients: a series report from multicenters. J Thorac Dis 2015;7:834-42.

18. Gonzalez-Rivas D, Fernandez R, Fieira E, et al. Uniportal video-assisted thoracoscopic bronchial sleeve lobectomy:First report. J Thorac Cardiovasc Surg 2013;145:1676-7.

19. He J, Wang W, Li J, et al. Video-assisted thoracoscopic surgery tracheal resection and carinal reconstruction for tracheal adenoid cystic carcinoma. J Thorac Dis 2016;8:198-203.

doi: $10.21037 /$ ccts-20-85

Cite this article as: Jia Z, Zhou W, Li S, Zhang Y, Zhang G, $\mathrm{Fu}$ J. Video-assisted thoracoscopic right upper sleeve lobectomy after neoadjuvant chemotherapy for central non-small cell lung cancer. Curr Chall Thorac Surg 2021;3:24.
20. Ludwig C, Engel-Riedel W, Stoelben E. Morbidity and mortality after neoadjuvant therapy and sleeve lobectomyin N2-disease. Zhongguo Fei Ai Za Zhi 2008;11:668-71.

21. Kim HK, Cho JH, Choi YS, et al. Outcomes of neoadjuvant concurrent chemoradiotherapy followed by surgery for non-small-cell lung cancer with N2 disease. Lung Cancer 2016;96:56-62.

22. Hirji SA, Osho A, Balderson SS, et al. Thoracoscopic lobectomy after induction therapy-a paradigm shift? J Vis Surg 2017;3:189.

23. Ismail M, Nachira D, Swierzy M, et al. Uniportal video-assisted thoracoscopy major lung resections after neoadjuvant chemotherapy. J Thorac Dis 2018;10:S3655-61.

24. Gonzalez M, Litzistorf Y, Krueger T, et al. Impact of induction therapy on airway complications after sleeve lobectomy for lung cancer. Ann Thorac Surg 2013;96:247-52.

25. Rea F, Marulli G, Schiavon M, et al. A quarter of a century experience with sleeve lobectomy for non-small cell lung cancer. Eur J Cardiothorac Surg 2008;34:488-92.

26. Storelli E, Tutic M, Kestenholz P, et al. Sleeve resections with unprotected bronchial anastomoses are safe even after neoadjuvant therapy. Eur J Cardiothorac Surg 2012;42:77-81.

27. Yildizeli B, Fadel E, Mussot S, et al. Morbidity, mortality, and long-term survival after sleeve lobectomy for non-small cell lung cancer. Eur J Cardiothorac Surg 2007;31:95-102. 